\title{
Demographic Characteristics of Sexually Transmitted Infections: Retrospective 5-year Outpatient Clinic Evaluation
}

\author{
(D) Gürkan Yardımcı ${ }^{1}$, (1) Server Serdaroğlu \\ ${ }^{1}$ Istanbul Medipol University, Esenler Hospital, Clinic of Dermatology, Istanbul, Turkey \\ 2Istanbul University Cerrahpasa-Cerrahpasa Faculty of Medicine, Department of Dermatology and Venereology, Istanbul, Turkey
}

\section{ABSTRACT}

Background: Sexually transmitted infections (STIS) are a health problem that can affect both genders and they continue to be a social problem. Although it is more common in young adults, it can affect individuals of any age. The aim of our study was to evaluate the demographic characteristics of STIs.

Materials and Methods: We investigated the cases of anogenital warts, herpes genitalis, genital molluscum contagiosum, syphilis, granuloma inguinale, lymphogranuloma venereum, pediculosis pubis and ulcus molle in 63,206 patients who applied to the outpatient clinic of the Dermatology Department of Istanbul University Cerrahpasa-Cerrahpasa Faculty of Medicine between 01.11.2007 and 01.12.2011.

Results: Anogenital warts was the most common disease. No records of patients diagnosed with granuloma inguinale, lymphogranuloma venereum, pediculosis pubis and ulcus molle were found. Although we found pediatric and elderly patient records in our search, most of the patients were young adults. According to the evaluation of gender distribution, no statistically significant difference was found except for anogenital warts.

Conclusion: In this retrospective study, we evaluated the anogenital warts, herpes genitalis, genital molluscum contagiosum, syphilis, granuloma inguinale, lymphogranuloma venereum, pediculosis pubis and ulcus molle patients that applied to our dermatology outpatient clinic; the parameters were the disease frequency, age, age distribution, gender and gender distribution by age. The frequency of anogenital warts, herpes genitalis, genital molluscum contagiosum and syphilis were $0.46 \%, 0.069 \%, 0.037 \%$ and $0.026 \%$, respectively. The average ages of these diseases were $34.58 \pm 12.64,33.3 \pm 14.91,20.29 \pm 12.24$, and $43.82 \pm 14.16$, respectively. While men were more affected by anogenital warts and syphilis, women were slightly more affected by genital molluscum contagiosum. The results were based upon the retrospective evaluation of the patients that applied to the outpatient clinic of Istanbul University Cerrahpasa-Cerrahpasa Faculty of Medicine Dermatology Department. Further studies with longer follow up intervals and larger case series are needed to reach nation-based conclusions.

Keywords: Anogenital warts, Demographic, Herpes genitalis, Molluscum contagiosum, Sexually transmitted infections, Syphilis

\section{Introduction}

Sexually transmitted infections (STIS) are caused by the transmission of various bacteria, viruses and ectoparasites from one person to another as a result of sexual contact [1]. According to The Centers for Disease Control and Prevention, it is estimated that there are close to 20 million new STIs each year in the United States (US). In addition, approximately half of these 20 million new cases are thought to be between the ages of 15-24 years [2]. Patients diagnosed with STIs can apply to urology and gynecology outpatient clinics as well as dermatology clinics. It was reported that more than 300,000 cases were diagnosed STI in genitourinary medicine clinics in England in 1996 [3]. In this study, we aimed to investigate the demographic 
characteristics of those diagnosed with STI among the patients who applied to the dermatology outpatient clinic.

\section{Materials and Methods}

In this study, we retrospectively investigated the cases of anogenital warts, herpes genitalis, genital molluscum contagiosum, syphilis, granuloma inguinale, lymphogranuloma venereum, pediculosis pubis and ulcus molle in total of 63,206 patients who applied to the outpatient clinic of the Dermatology Department of Istanbul University Cerrahpasa-Cerrahpasa Faculty of Medicine between 01.11.2007-01.12.2011. Approval of the Cerrahpasa Medical Faculty Ethics Committee was taken (13.03.2012-B.30.2.IST.0.30.11.06/109).

The diagnosis of anogenital warts, herpes genitalis and genital molluscum contagiosum were made by detailed dermatologic and genital examination. In suspected herpes genitalis cases, the diagnosis was confirmed by herpes simplex virus immunoglobulin $\mathrm{G}$ antibody positivity. The diagnosis of syphilis was made by performing serological tests.

In this study; frequency, age, gender and gender distribution by age were taken into account in all patients.

\section{Statistical Analysis}

The data were recorded in a computer data entry program based on the International Statistical Classification of Diseases and Related Health Problems (International Classification of Diseases) diagnostic code system and were retrospectively reviewed. The data were evaluated by statistical study.

Statistical analyzes were performed using Statistical Package for the Social Sciences V.15.0 and Number Cruncher Statistical System 2007 programs. $\mathrm{P}<0.05$ value was considered statistically significant. Chisquare test was applied in the analyzes and frequency tables and descriptive statistics were used.

\section{Results}

Although anogenital warts, herpes genitalis, genital molluscum contagiosum, syphilis, granuloma inguinale, lymphogranuloma venereum, pediculosis pubis and ulcus molle among the STIs were included in the screening, no record of four diseases (granuloma inguinale, Iymphogranuloma venereum, pediculosis pubis and ulcus molle) could be found.

In this section, the findings were evaluated separately for anogenital warts, herpes genitalis, genital molluscum contagiosum and syphilis.

\section{Anogenital Warts}

A total of 63,206 patients who admitted to Istanbul University Cerrahpasa-Cerrahpasa Faculty of Medicine Dermatology Department between 01.11.2007-31.12.2011, 297 (0.46\%) of them were diagnosed with anogenital warts. Of the 297 patients diagnosed, 231 (77.8\%) of them were male and 66 (22.2\%) of them were female. Anogenital warts were more common in men than in women and this difference was found to be statistically significant $(p<0.001)$. The mean age of the patients was 34.58 \pm 12.64 (3-88 years) years. These patients with the diagnosis of anogenital warts were divided into nine groups according to their age. The incidence of the disease showed statistically significant difference among age groups $(p<0.001)$. The age group with the highest frequency was the 30 -39 age group (34.3\%) and the second most frequent was the 2029 age group (31.3\%). There was a statistically significant difference among age groups in terms of gender $(p<0.001)$. While the disease was most common (36.4\%) in the 30-39 age group in men, it was most common (36.4\%) in the 20-29 age group in women. Of the 81.8\% diagnosed patients with anogenital warts were found between the ages of 20-49 years. Gender distribution of patients with anogenital warts according to age groups is shown in Table 1.

\section{Herpes Genitalis}

Between the dates 01.11.2007-31.12.2011, out of 63,206 patients who applied to Cerrahpasa Medical Faculty Dermatology Department, $44(0.069 \%)$ of them were diagnosed with herpes genitalis. Of the 44 patients, 22 (50\%) of them were male and 22 (50\%) of them were female. There was no statistically significant difference between male and female distribution $(p=1.000)$. The mean age of the patients was 33.3 \pm 14.91 (1-68 years) years. These patients with the diagnosis of herpes genitalis were divided into seven groups according to their age. The incidence of the disease showed statistically significant difference among age groups $(p=0.003)$. The age group with the highest frequency was the 30 39 age group (27.3\%) and the second most frequent was the 2029 age group (25\%). There was no statistically significant difference among age groups in terms of gender $(p=0.436)$. While the disease was most common (36.4\%) in the 30-39 age group in men, it was most common (31.8\%) in the 20-29 age group in women. Of the $75 \%$ diagnosed patients with herpes genitalis were found between the ages of 20-49 years. Gender distribution of patients with herpes genitalis according to age groups is shown in Table 2.

\section{Genital Molluscum Contagiosum}

Between the dates 01.11.2007-31.12.2011, out of 63,206 patients who applied to Cerrahpasa Medical Faculty Dermatology Department, $24(0.037 \%)$ of them were diagnosed with genital molluscum contagiosum. Of the 24 patients, 10 (41.7\%) of them were male and 14 (58.3\%) of them were female. There was no statistically significant difference between male and female distribution $(p=0.414)$. The mean age of the patients was 20.29 \pm 12.24 (3-48 years) years. These patients with the diagnosis of genital molluscum contagiosum were divided into five groups according to their age. The incidence of the disease showed statistically significant difference among age 
groups ( $p=0,002)$. The age group with the highest frequency was the 20-29 age group (45.8\%) and the second most frequency was 0-9 age group (33.3\%). There was no statistically significant difference among age groups in terms of gender $(p=0.329)$. The disease was most frequently observed in the 20-29 age group in both gender (male: $40 \%$, female: $50 \%$ ). Out of 24 patients, 11 (45.8\%) of them was

\section{Table 1. Gender distribution of patients with anogenital warts according to age groups}

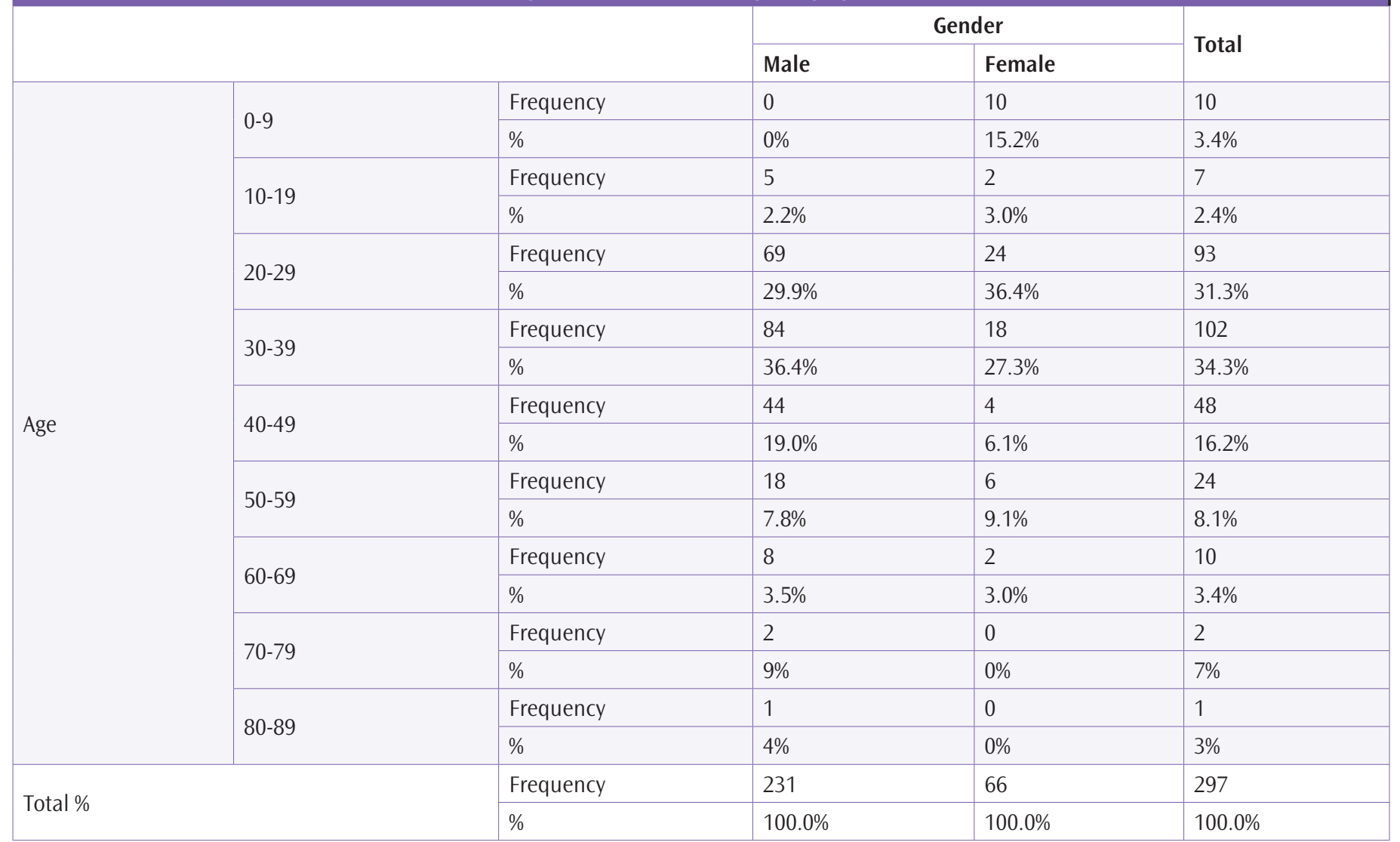

\section{Table 2. Gender distribution of patients with herpes genitalis according to age groups}

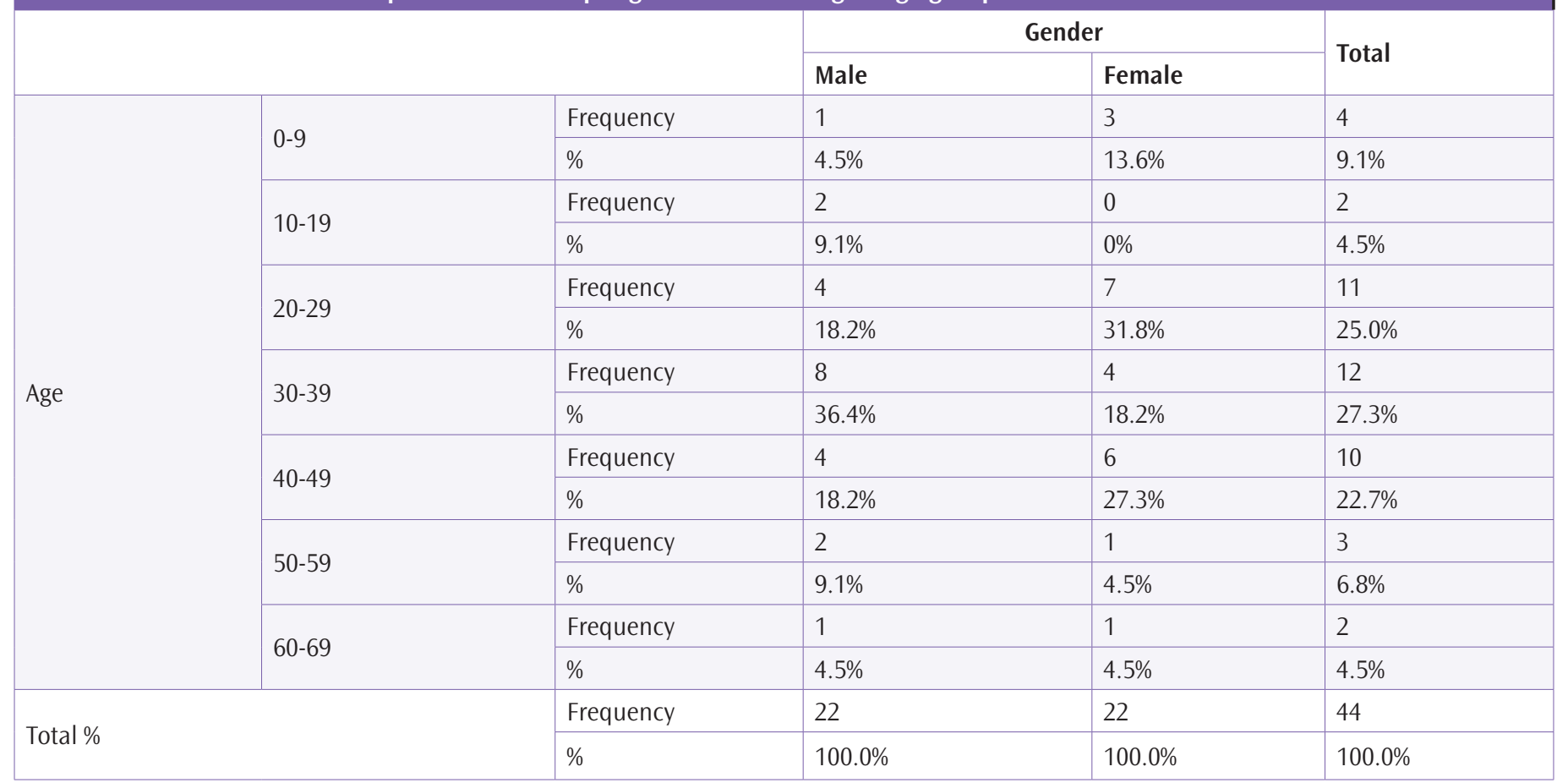


found between the ages of 20-29. Gender distribution of patients with genital molluscum contagiosum according to age groups is shown in Table 3.

\section{Syphilis}

Between the dates 01.11.2007-31.12.2011, out of 63,206 patients who applied to Cerrahpasa Medical Faculty Dermatology Department, $17(0.026 \%)$ of them were diagnosed with syphilis. Out of 17 patients, 10 (58.8\%) of them were male and 7 (41.2\%) of them were female. There was no statistically significant difference between male and female distribution $(p=0.466)$. The mean age of the patients was $43.82 \pm 14.16$ (20-78 years) years. These patients with the diagnosis of syphilis were divided into six groups according to their age. The incidence of the disease showed statistically significant difference among age groups $(p=0.017)$. The age group with the highest frequency was the 30-39 age group (47.1\%) and the second most frequency was 50-59 age group (23.5\%). There was no statistically significant difference among age groups in terms of gender $(p=0.342)$. While the disease was most common $(60 \%)$ in the 30-39 age group in men, it was most common (42.9\%) in the 50-59 age group in women. Out of 17 patients, 14 (82.4\%) of them were found between the ages of 30-59 years. Gender distribution of patients with syphilis according to age groups is shown in Table 4.

\section{Discussion}

STIs continue to be a serious public health problem worldwide for years [4]. It is estimated that there are approximately 19 million

Table 3. Gender distribution of patients with genital molluscum contagiosum according to age groups

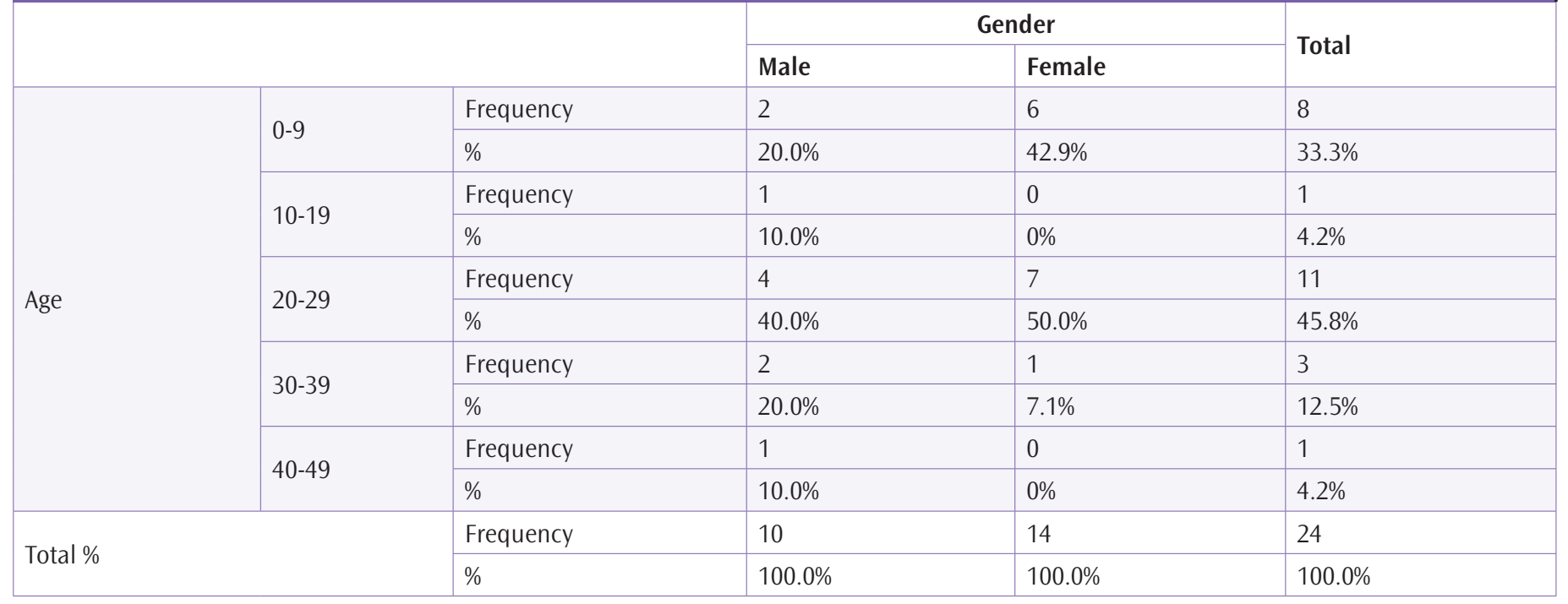

Table 4. Gender distribution of patients with syphilis according to age groups

\begin{tabular}{|c|c|c|c|c|c|}
\hline & & & \multicolumn{2}{|c|}{ Gender } & \multirow{2}{*}{ Total } \\
\hline & & & Male & Female & \\
\hline \multirow{12}{*}{ Age } & \multirow{2}{*}{$20-29$} & Frequency & 0 & 1 & 1 \\
\hline & & $\%$ & $0 \%$ & $14.3 \%$ & $5.9 \%$ \\
\hline & \multirow{2}{*}{$30-39$} & Frequency & 6 & 2 & 8 \\
\hline & & $\%$ & $60.0 \%$ & $28.6 \%$ & $47.1 \%$ \\
\hline & \multirow{2}{*}{$40-49$} & Frequency & 1 & 1 & 2 \\
\hline & & $\%$ & $10.0 \%$ & $14.3 \%$ & $11.8 \%$ \\
\hline & \multirow{2}{*}{ 50-59 } & Frequency & 1 & 3 & 4 \\
\hline & & $\%$ & $10.0 \%$ & $42.9 \%$ & $23.5 \%$ \\
\hline & \multirow{2}{*}{$60-69$} & Frequency & 1 & 0 & 1 \\
\hline & & $\%$ & $10.0 \%$ & $0 \%$ & $5.9 \%$ \\
\hline & \multirow{2}{*}{$70-79$} & Frequency & 1 & 0 & 1 \\
\hline & & $\%$ & $10.0 \%$ & $0 \%$ & $5.9 \%$ \\
\hline \multirow{2}{*}{\multicolumn{2}{|c|}{ Total \% }} & Frequency & 10 & 7 & 17 \\
\hline & & $\%$ & $100.0 \%$ & $100.0 \%$ & $100.0 \%$ \\
\hline
\end{tabular}


new STI cases in the US and approximately 300 million new STI cases in the world every year [2,5]. Similarly, the incidence of STIS in developed countries of Europe has been increasing for the last 20 years [6].

Although adolescents and young adults are the high-risk group for STIs, it is important to consider the possible risk of STIs in the elderly population as well. Most elderly patients continue to have heterosexual or homosexual relationships despite their advancing age [7]. About half of the new cases observed each year in the US are composed of young people aged 15-24 years [8]. In our study, in accordance with the literature data, STIs rates were higher in young adults than in the elderly population.

Human papilloma virus (HPV) infections are the most commonly diagnosed STI [9]. In our study, the most frequently diagnosed STI among the diseases we screened was anogenital warts. Of the 63,206 patients included in our study, 297 (0.46\%) of them were diagnosed with anogenital warts. The data we obtained in terms of frequency were found to be lower than we expected. This may be due to the fact that male and female patients refer to the non-dermatology clinics, such as urology and the gynecology clinic. Other possible reasons for the lower frequency of the disease than we expected could be that Turkish individuals felt themselves under public pressure in terms of diseases affecting the genital area, and thus did not apply to the hospital due to a sense of shyness or were seeking a cure in private medical centers. According to the English literature, sexually active women aged $<25$ years have the highest rates of genital HPV infections [9]. But in a study from Turkey, $84.3 \%$ and $15.7 \%$ of 83 patients with anogenital warts were reported as male and female, respectively [10]. The US National Health and Nutrition Examination Survey reported the overall prevalence of HPV infection in women aged 14-59 years as $26.8 \%$. The rates of prevalence of HPV infection were reported as $44.8 \%, 27.4 \%$ and $24.5 \%$ in women aged $20-24$ years, 25-29 years, and 14-19 years, respectively [9]. The prevalence of genital warts in men was most commonly reported between the ages of 25-29 years by Insinga et al. [11]. However, our data were different in terms of gender compared to the English literature. Anogenital warts, which are more common in women in general, were found to be lower in our study $(77.8 \%$ male and $22.2 \%$ female). The mean age of the patients was 34.58 \pm 12.64 (3-88) years. Similar to literature data, the most common age range of our female patients was found to be $20-29$ years (36.4\%). But, the majority of the male group was between the ages of 30-39 years (36.4\%), and these data were different from the literature. The fact that $81.8 \%$ of patients diagnosed with anogenital warts were between the ages of 20-49 supported that the disease is common in young adults.
Although the seroprevalence of HSV-2 in the US is approximately $20 \%$, it has been reported that herpes genitalis cases due to HSV1 , especially at the adolescent age, have increased in recent years $[12,13]$. There are also some studies in the literature that herpes genitalis is more common in women than in men $[14,15]$. In our study, $0.069 \%$ of all patients were diagnosed with herpes genitalis and the numbers of male and female patients were equally distributed ( $50 \%$ male and $50 \%$ female). The mean age of the patients was $33.3 \pm 14.91$ (1-68) years. The age group with the highest frequency was the 30-39 age group (27.3\%) and the second most frequent was the 20-29 age group (25\%). Most patients, $75 \%$ of all patients with herpes genitalis, were between the ages of 20-49 years and this data was found to be consistent with the information that the disease is more common in young adults. We believe that herpes genitalis is more common in the population. Similar to patients with anogenital warts, female patients applying to the gynecology outpatient clinic may be a reason for reducing the number of applications to the dermatology outpatient clinic. In addition, recurrent attacks of herpes genitalis can be neglected by the patients because they are mildly symptomatic or asymptomatic.

We could not found much data in the literature on the frequency of genital molluscum contagiosum. In our study, 24 patients $(0.037 \%$ of all patients) were diagnosed with genital molluscum contagiosum. Although it is slightly more common in women in terms of gender, a statistically significant difference was found. The mean age of the patients was $20.29 \pm 12.24$ (3-48) years. Although the age group with the highest frequency was the $20-29$ age group (45.8\%), it was remarkable that the 0-9 age group was the second most frequent (33.3\%).

Although there is a periodic increase in the frequency of syphilis, there has been a decrease in general in recent years [16]. We found the frequency of syphilis as $0.026 \%$. Although we could not find a statistically significant difference, we found that it was more common in men (58.8\%) than in women (41.2\%). According to the data reported from Turkey and abroad in recent years, it has been reported that it is more common in men than in women $[17,18]$. Our data can also support the increase in the male:female ratio reported in recent years. Although the mean age of the patients was $43.82 \pm 14.16$ (20-78) years, the age group with the highest frequency was the 30-39 age group (47.1\%). More than $80 \%$ of the patients diagnosed with syphilis were between the ages of 30-59, so syphilis was more common in adults, consistent with our other diseases. As a result of our observations, most of the patients who were diagnosed with syphilis were identified after an incidental syphilis serology. This situation can be interpreted as the patients did not apply to the hospital in the primary and secondary stages. 
The fact that granuloma inguinale, lymphogranuloma venereum, pediculosis pubis and ulcus molle, among other diseases included in our study, could not be detected in any of the patients suggests that these diseases are not common in our country. However, the possibility that these rare diseases can be overlooked by inexperienced physicians and can easily miss the diagnosis, as well as the fact that some of the laboratory tests required to confirm the diagnosis are not available in our hospital may be the reasons for not being able to diagnose these diseases.

Other possible reasons for the low frequency of all diseases are i) the patient registration and follow-up system does not work properly, ii) since our hospital is a university hospital, our department is not seen as the first point of application by patients, iii) as a result of the fact that patients are psychologically affected by their illness and they perceive it as a feeling of guilt, they either never apply to the hospital or they apply to a private hospital/medical center because their personal information is requested to be kept confidential.

\section{Study Limitations}

The results were based upon the retrospective evaluation of the patients that applied to the outpatient clinic of Istanbul University Cerrahpasa-Cerrahpasa Faculty of Medicine Dermatology Department. Further studies with longer follow up intervals and larger case series are needed to reach nation-based conclusions.

\section{Conclusion}

In this retrospective study, we evaluated the anogenital warts, herpes genitalis, genital molluscum contagiosum, syphilis, granuloma inguinale, lymphogranuloma venereum, pediculosis pubis and ulcus molle patients that applied to our dermatology outpatient clinic; the parameters were the disease frequency, age, age distribution, gender and gender distribution by age.

The frequency of anogenital warts, herpes genitalis, genital molluscum contagiosum and syphilis were 0.46\%, 0.069\%, 0.037\% and $0.026 \%$, respectively. The average ages of these diseases were $34.58 \pm 12.64,33.3 \pm 14.91,20.29 \pm 12.24$, and $43.82 \pm 14.16$, respectively. Although the frequency data were low as we thought, most of the data related to age were found to be compatible with the literature data.

In terms of gender distribution; while men were more affected by anogenital warts and syphilis, women were slightly more affected by genital molluscum contagiosum. However, the gender distribution in herpes genitalis was equal. According to the evaluation of these data, no statistically significant difference was found except for anogenital warts.

No records of patients diagnosed with granuloma inguinale, lymphogranuloma venereum, pediculosis pubis and ulcus molle were found.
Larger case series and multi-center studies, including patients admitted to urology and gynecology outpatient clinics, are needed in order to interpret the data more accurately on demographic characteristics and frequencies of STIS.

Ethics

Ethics Committee Approval: Approval of the Cerrahpasa Medical Faculty Ethics Committee was taken (13.03.2012-B.30.2.I ST.0.30.11.06/109).

Informed Consent: Retrospective study.

Peer-review: Externally and internally peer-reviewed.

\section{Authorship Contributions}

Surgical and Medical Practices: G.Y., Concept: G.Y., S.S., Design: G.Y., S.S., Data Collection or Processing: G.Y., S.S., Analysis or Interpretation: G.Y., S.S., Literature Search: G.Y., Writing: G.Y.

Conflict of Interest: No conflict of interest was declared by the authors.

Financial Disclosure: The authors declared that this study received no financial support.

\section{References}

1. Wagenlehner FM, Brockmeyer NH, Discher T, Friese K, Wichelhaus TA. The Presentation, Diagnosis, and Treatment of Sexually Transmitted Infections. Dtsch Arztebl Int 2016;113:11-22.

2. Brill JR. Sexually transmitted infections in men. Prim Care 2010;37:509-525.

3. Carne C. Sexually transmitted infections. BMJ 1998;317:129-132.

4. de Amorim TF, Teles SA, Moraes LC, de Matos MA, Carneiro MADS, Nogueira DJ, Rosa LRDC, E Silva GRDC, Caetano KAA. Symptomatic Sexually Transmitted Infections in Brazil's Emerging Rural Populations. J Assoc Nurses AIDS Care 2018;29:942-948.

5. Choe HS, Lee SJ, Kim CS, Cho YH. Prevalence of sexually transmitted infections and the sexual behavior of elderly people presenting to health examination centers in Korea. J Infect Chemother 2011;17:456-461.

6. Boffin N, Moreels S, Deblonde J, Van Casteren V. Four sexually transmitted infections (STIS) in Belgian general practice: first results (2013-2014) of a nationwide continuing surveillance study. BMJ Open 2017;7:e012118.

7. Rosen T, Brown TJ. Cutaneous manifestations of sexually transmitted diseases. Med Clin North Am 1998;82:1081-1104.

8. Gibson EJ, Bell DL, Powerful SA. Common sexually transmitted infections in adolescents. Prim Care 2014;41:631-650.

9. Steben M, Duarte-Franco E. Human papillomavirus infection: epidemiology and pathophysiology. Gynecol Oncol 2007;107:S2-S5.

10. Serdaroğlu S, Akkurt M, Kușkucu M, Midilli K, Bahçetepe N, Yılmaz G. The Determination of HPV Types in Anogenital Warts and Research of it's Relationship with Recurrence. Dermatoz 2010;1;173-176.

11. Insinga RP, Dasbach EJ, Myers ER. The health and economic burden of genital warts in a set of private health plans in the United States. Clin Infect Dis 2003;36:1397-1403.

12. Lee AJ, Ashkar AA. Herpes simplex virus-2 in the genital mucosa: insights into the mucosal host response and vaccine development. Curr Opin Infect Dis 2012;25:92-99 
13. Gardella C, Brown ZA. Managing genital herpes infections in pregnancy. Cleve Clin J Med 2007;74:217-224.

14. Westhoff GL, Little SE, Caughey AB. Herpes simplex virus and pregnancy: a review of the management of antenatal and peripartum herpes infections. Obstet Gynecol Surv 2011;66:629-638.

15. Gupta R, Warren T, Wald A. Genital herpes. Lancet 2007;370:2127-2137.
16. Little JW. Syphilis: an update. Oral Surg Oral Med Oral Pathol Oral Radiol Endod 2005;100:3-9.

17. Zeltser R, Kurban AK. Syphilis. Clin Dermatol 2004;22:461-468.

18. Apaydin R, Bilen NG, Gül U, Bahadir S. Increased number of the cases of syphilis in Trabzon, a trade city in the Black Sea region of Turkey. Sex Transm Infect 1998;74:377. 